\title{
INTEGRATION IS DIFFERENTIABLE ${ }^{1}$
}

\author{
ALLAN GOTTLIEB
}

ABSTRACT. The integration map taking vector fields to flows is well known to be continuous. In this paper an elementary proof is given that the map is actually smooth if the domain and range are given appropriate topologies.

In the elementary theory of ordinary differential equations, one shows that, under suitable hypotheses, the solution depends differentiably on the initial condition and continuously on the original differential equation. The major result of this paper is an elementary proof that, by choosing appropriate topologies, the latter dependency is also smooth.

If $M$ is a closed (i.e. compact boundaryless) $C^{r}$ manifold and $X$ is a $C^{r}$ vectorfield on $M(r \geq 1), X$ induces $\Psi^{X}$, a one parameter group of diffeomorphisms or flow, $\Psi^{X}: M \times \mathbf{R} \rightarrow M$. For $p \in M$ fixed, the curves $t \mapsto \Psi^{X}(p, t)$ are called integral curves and the map, $\Psi$, taking $X$ to $\Psi^{X}$, is often called integration.

If $A$ and $B$ are boundaryless manifolds, denote by $C^{r}(A, B)$ the $C^{r}$ maps from $A$ to $B$, and if $E$ is a $C^{r}$ vector bundle, denote by $C^{r}(E)$ the $C^{r}$ cross sections. In particular, $C^{r}(T M)$ is the space of $C^{r}$ vectorfields on $M$. Using this notation:

$$
\begin{aligned}
\Psi: C^{r}(T M) & \rightarrow C^{r}(M \times \mathbf{R}, M), \quad r \geq 1, \\
X & \mapsto \Psi^{X} .
\end{aligned}
$$

Let $J=[a, b] \subset \mathbf{R}$ be a compact interval. Denote by $\left.\Psi^{X}\right|_{M \times J}$ the restriction of $\Psi^{X}$ to $M \times J$. Abusing notation we define

$$
\Psi: C^{r}(T M) \rightarrow C^{r}(M \times J, M), \quad r \geq 1, \quad \text { by } \Psi(X)=\left.\Psi^{X}\right|_{M \times J} .
$$

It is well known that this last map is continuous when $C^{r}(T M)$ is given the $C^{r}$ topology (see remarks below). We shall give an elementary proof of the following generalization.

Theorem. Let $M$ be a closed $C^{r+s}$ manifold with $r+s \geq 1$ and let $J$ be a compact interval. If $C^{r+s}(T M)$ is given the $C^{r+s}$ norm and $C^{r}(M \times J, M)$ is given the $C^{r}$ topology, then the map $\Psi: C^{r+s}(T M) \rightarrow C^{r}(M \times J, M) \cdot d e$. fined by $\Psi(X)=\left.\Psi^{X}\right|_{M \times J}$ is $C^{s}$.

Received by the editors June 7, 1974 and, in revised form, September 9, 1974. AMS (MOS) subject classifications (1970). Primary 34A10, 34C40.

${ }^{1}$ Research partially supported by CUNY Faculty Research Award Program Grant $10272 \mathrm{~N}$. 
Remarks. (1) The case $s=0$ is the above well-known result (in that case one need not restrict to $M \times J$ ) and Robinson, using different techniques, has independently proved the case $s=1$ [4].

(2) The boundary of $M \times J$ introduces a technical difficulty into defining $C^{r}(M \times J, M)$. However, the map $\Psi^{X}$ is $C^{r+s}$ on all of $M \times \mathbf{R}$, so the above causes no real problem.

Proof. Consider the $C^{r+s}$ parameterized vectorfield (parameterized by $C^{r+s}(T M)$ ), ev: $C^{r+s}(T M) \times M \rightarrow T M$ defined by $\operatorname{ev}(X, p)=X(p)$ (ev stands for evaluation).

Note that for a fixed parameter value, $X_{0}$, the above becomes $p \rightarrow X_{0}(p)$, i.e., $X_{0}$ itself.'As $M$ is closed, we may apply the parameterized flow theorem [1] to obtain a $C^{r+s}$ parameterized flow $B: C^{r+s}(T M) \times M \times R \rightarrow M . B$ is $C^{r+s}$ as a map from the triple product and satisfies

$$
d B(X, p, t) / d t=\operatorname{ev}(X, B(X, p, t))=X(B(X, p, t)) .
$$

Thus, by the uniqueness theorem for solutions to ordinary differential equations, $B(X, p, t)=\Psi^{X}(p, t)$.

Restricting $B$ to $C^{r+s}(M) \times M \times J$ gives a $C^{r+s}$ map

$$
B: C^{r+s}(T M) \times(M \times J) \rightarrow M, \quad(X,(p, t)) \mapsto \Psi^{X}(p, t) .
$$

The Theorem now follows from

Lemma. Let $T$ be a Banach space (actually a $C^{r+s}$ Banach manifold would do) and let $X$ and $Y$ be closed $C^{r+s}$ manifolds. Assume that $F: T \times X \rightarrow Y$ is a $C^{r+s}$ map. Then the induced map $\vec{F}: T \rightarrow C^{r}(X, Y)$ is $C^{s}$.

Proof. Case 1. $r=s=0$.

This is an elementary result on metric spaces [2].

Case 2. The local case: $X=D^{n}$, the closed $n$-disc, and $Y$ an arbitrary Banach space.

Note that in Case 2, $C^{r}(X, Y)$ is a Banach space using the $C^{r}$ norm, i.e., the maximum of the norms of the function and its first $r$ derivatives.

It suffices to show that for $0 \leq j \leq s ; t, t_{1}, \ldots, t_{j} \in T$; and $x \in X ; D^{j} \bar{F}$ exists and

$$
D^{j} \bar{F}(t)\left(t_{1}\right) \cdots\left(t_{j}\right)(x)=D_{1}^{j} F(t, x)\left(t_{1}\right) \cdots\left(t_{j}\right)=D F(t, x)\left(t_{1}, 0\right) \cdots\left(t_{j}, 0\right) .
$$

The main tool for this is the

Converse to Taylor's Theorem [1]. Let $E$ and $V$ be Banach spaces and let $f: E \rightarrow V$. Assume that, for $0 \leq j \leq s$, we have a continuous map $\theta_{j}: E \rightarrow L_{s}^{j}(E, V)$ and define $\lambda_{:} E \times E \rightarrow V$ by

$$
f(x+h)=\sum_{j=0}^{s} \frac{\theta_{j}(x)(h)^{j}}{j !}+\lambda(x, h),
$$


where $(h)^{j}=(h) \ldots(h)$ ( $j$ occurrences) and $L_{s}^{j}(E, V)$ is the Banach space of symmetric $j$-multilinear maps from $E$ to $V$. Also assume that

$$
\|\lambda(x, h)\| /\|b\|^{s} \rightarrow 0 \text { as }(x, h) \rightarrow 0 .
$$

Then $f$ is $C^{s}$ and $D^{j} f=\theta_{j}(0 \leq j \leq s)$.

For $0 \leq j \leq s$, define $A_{j}: T \rightarrow L_{s}^{j}\left(T, C^{r}(X, Y)\right)$ by

$$
A_{j}(t)\left(t_{1}\right) \cdots\left(t_{j}\right)(x)=D_{1}^{j} F(t, x)\left(t_{1}\right) \cdots\left(t_{j}\right)=D^{j} F(t, x)\left(t_{1}, 0\right) \cdots\left(t_{j}, 0\right) .
$$

Let \|\|$_{r}$ be the $C^{r}$ norm on $C^{r}(X, Y)$, and for $0 \leq i \leq r$ let \|\|$_{i}$ be the operator norm on $L_{s}^{i}\left(R^{n}, Y\right)$. Since in Case $2 X=D^{n}$ is not a Banach space, $L_{s}^{i}(X, Y)$ does not make sense.

For Case 2 we need only verify (1) when $f=\bar{F}$ and $\theta_{j}=A_{j}$. The key is the following consequence of the definition of higher derivatives: for $0 \leq i \leq r$; $0 \leq j \leq s ; t, t_{1}, \ldots, t_{j} \in T ; x \in X$ and $x_{1}, \ldots, x_{j} \in X$

$$
\begin{aligned}
D^{i}\left(A_{j}(t)\left(t_{1}\right) \cdots\left(t_{j}\right)\right)(x)\left(x_{1}\right) \cdots\left(x_{i}\right) & \\
& =D^{i+j} F(t, x)\left(t_{1}, 0\right) \cdots\left(t_{j}, 0\right)\left(0, x_{1}\right) \cdots\left(0, x_{i}\right) .
\end{aligned}
$$

To estimate the remainder $\lambda$ needed for (1), fix $t \in T$ and $\epsilon>0$. For $b \in T$ we have

$$
\begin{gathered}
\left\|\bar{F}(t+h)-\sum_{j=0}^{s} \frac{A_{j}(t)(h)^{j}}{j !}\right\|=\sup _{0 \leq i \leq r ; x \in X}\left\|D^{i}(\bar{F}(t+h))(x)-\sum_{j=0}^{s} \frac{D^{i}\left(A_{j}(t)(h)^{i}\right)(x)}{j !}\right\|_{i} \\
=\sup _{\substack{0 \leq i \leq r ; x \in X \\
k_{1}, \cdots, k_{i} \in R^{n}}}\left\|D^{i(}(\bar{F}(t+h))(x)\left(k_{1}\right) \cdots\left(k_{i}\right)-\sum_{j=0}^{s} \frac{D^{i}\left(A_{j}(t)(h)^{j}\right)(x)\left(k_{1}\right) \cdots\left(k_{i}\right)}{j !}\right\| . \\
\left\|k_{1}\right\|=\cdots=\left\|k_{i}\right\|=1
\end{gathered}
$$

Using (2) and interchanging the order of differentiation, the above becomes

$\sup \left\|D^{i} F(t+h, x)\left(0, k_{1}\right) \cdots\left(0, k_{i}\right)-\sum_{j=0}^{s} \frac{D^{i+j} F(t, x)\left(0, k_{1}\right) \cdots\left(0, k_{i}\right)(h, 0)^{j}}{j !}\right\|$.

Let $Z_{i}=D^{n} \times\left(S^{n-1}\right)^{i}$ and let $v=\left(x, k_{1}, \ldots, k_{i}\right) \in Z$. Define $G^{v}: T \rightarrow Y$ by $G^{v}(\bar{t})=D^{i} F(\bar{t}, x)\left(0, k_{1}\right) \ldots\left(0, k_{i}\right)$. Using this new notation and again jiggling the order of differentiation, (3) becomes

$$
\sup _{0 \leq i \leq r ; v \in Z_{i}}\left\|G^{v}(t+h)-\sum_{j=0}^{s} \frac{D^{j} G^{v}(t)(h)^{j}}{j !}\right\|
$$

Finally define $R_{i}: Z \times T \rightarrow R$ by $R_{i}(\nu, 0)=0$ and, for $h \neq 0$, 


$$
R_{i}(v, h)=\left\|G^{\nu}(t+h)-\sum_{j=0}^{s} \frac{D^{j} G^{\nu}(t)(h)^{j}}{j !}\right\| /\|h\|^{s} .
$$

By Taylor's theorem, $R_{j}$ is continuous at $(v, 0)$ for all $v \in Z$. Since $R_{i}(v, 0)=0, Z$ is compact, and $i$ is restricted to a finite set; there exists $\delta>0$ such that for $0 \leq i<r, v \in Z$, and $\|h\|<\delta$,

$$
\left\|R_{i}(v, h)\right\|<\epsilon \text {. }
$$

But tracing back through the myriad of definitions, one finds that (4) is just (1) as desired.

Case 3. Semilocal case, $X=D^{n}$.

For $t \in T, \bar{F}(t): X \rightarrow Y$. A $C^{0}$ neighborhood $U$ of $\bar{F}(t) \in C^{r}(X, Y)$ is isomorphic to a neighborhood of zero in $C^{r}\left((\bar{F}(t))^{*}(T Y)\right)$. But this space is isomorphic to $C^{r}\left(D^{n}, R^{n}\right)$ since $X=D^{n}$ is contractible. By Case 1 , for $t^{\prime}$ near $t, \bar{F}\left(t^{\prime}\right) \in U$. Thus Case 3 is reduced to Case 2 .

Case 4. General case.

Write $X$ as the finite union of $n$-discs $X=\bigcup_{i}^{k} D_{i}^{n}$, and let

$$
F_{i}=\left.F\right|_{T \times D_{i}^{n:}} T \times D_{i}^{n} \rightarrow Y .
$$

By Case 3, $\bar{F}_{i}: T \rightarrow D^{r}\left(D_{i}^{n}, Y\right)$ is $C^{s}$. In [3, Theorem A4, p. 74] I gave a brute force, rather boring, proof that the above suffices for Case 4 . The following more elegant proof is undoubtedly better.

Sublemma. The map

$$
J: C^{r}(X, Y) \rightarrow C^{r}\left(D_{1}^{n}, Y\right) \times \cdots \times C^{r}\left(D_{k}^{n}, Y\right), \quad f \rightarrow\left(\left.f\right|_{D_{1}^{n^{2}}}, \cdots,\left.f\right|_{D_{k}^{n^{n}}}\right)
$$

is a smooth imbedding:

As $J \circ \bar{F}=\bar{F}_{i}$ is $C^{s}$ by Case 3, Case 4 follows from the Sublemma.

Proof of the Sublemma. We must show that, for all $f \in C^{r}(X, Y)$, the tangent map $T J(f)$, is split injective. This will be accomplished by using a partition of unity to explicitly construct the required left inverse.

The tangent space at $f$ to $C^{r}(X, Y),\left(C^{r}(X, Y)\right)_{f}$, is the space of all "sections" $\sigma$ covering $f$, i.e., commuting the diagram

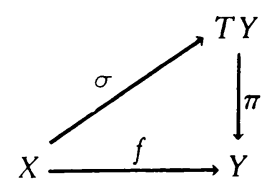

where $\pi$ is the canonical projection (note that $X$, as well as each $D_{i}^{n}$, is compact). For $h \in C^{r}\left(D_{i}^{n}, Y\right)$, the definition of $\left(C^{r}\left(D_{i}^{n}, Y\right)\right)_{h}$ is similar.

It is clear that, for $\sigma \in\left(C^{r}(X, Y)\right)_{f}$, 


$$
T J(f)(\sigma)=\left(\left.\sigma\right|_{D_{1}^{n}}, \ldots,\left.\sigma\right|_{D_{1}^{n}}\right) \in\left(C^{r}\left(D_{1}^{n}, Y\right)\right)_{f_{1}} \times \cdots \times\left(C^{r}\left(D_{k}^{n}, Y\right)\right)_{f_{k}}
$$

where $f_{i}=\left.f\right|_{D_{i}^{n}}$.

Choose a smooth partition of unity, $\left\{\phi_{i}\right\}$, subordinate to the cover $\left\{D_{i}^{n}\right\}$, i.e.

(1) $\phi_{i}: X \rightarrow[0,1]$,

(2) $\phi_{i}$ has support contained in $D_{i}^{n}$,

(3) $\Sigma_{i} \phi_{i}(x)=1$, for all $x \in X$.

Finally, define the map

$$
L:\left(C^{r}\left(D_{1}^{n}, Y\right)\right)_{f_{1}} \times \cdots \times\left(C^{r}\left(D_{k}^{n}, Y\right)\right)_{f_{k}} \rightarrow\left(C^{r}(X, Y)\right)_{f}
$$

by

$$
L\left(\sigma_{1}, \ldots, \sigma_{k}\right)(x)=\sum_{i} \phi_{i}(X) \sigma_{i}(X) .
$$

It is easy to see that $(L \circ T J(f))(\sigma)=\sigma$ so $L$ is the required left inverse.

\section{REFERENCES}

1. R. Abraham and J. Robbin, Transversal mappings and flows, Benjamin, New York, 1967. MR $39 \# 2181$.

2. J. Dugundji, Topology, Allyn and Bacon, Boston, Mass., 1966. MR 33 \#1824.

3. A. Gottlieb, Thesis, Brandeis University, Waltham, Mass., 1973.

4. C. Robinson (unpublished).

DEPARTMENT OF MATHEMATICS, YORK COLLEGE, CITY UNIVERSITY OF NEW YORK, JAMAICA, NEW YORK 11432 